\title{
MORPHOLOGY BASED TECHNIQUE FOR TEXTURE ENHANCEMENT AND SEGMENTATION
}

\author{
M.Joseph Prakash ${ }^{1}$, Dr.V.Vijaya Kumar ${ }^{2} \&$ Dr.A.VinayaBabu ${ }^{3}$ \\ ${ }^{1}$ Research Scholar, JNTUK University, Kakinada \\ mojesphp7@gmail.com \\ ${ }^{2}$ Department of CSE, GIET, JNTUK University, Rajahmundry, India \\ vijayavakula@yahoo.com \\ ${ }^{3}$ Department of CSE, JNTUHCE, JNTUH University, Hyderabad, India \\ avbb1222@gmail.com
}

\begin{abstract}
In the field of image analysis 'texture' plays a crucial role. A texture can be thougt of as an ensemble of repetitive sub patterns, which follow a group of pre- defined arrangement rules. The goal of image segmentation is to detect and extract the regions, which compose an image. In image analysis, understanding and interpretation texture segmentation is one of the most important techniques. The goal of texture segmentation is to divide the image into a group of regions such that each region has the same textural properties. The present paper proposes a new segmentation method for noise removal, image enhancement and segmentation. The proposed algorithm offers the advantage of providing good quality segmentation. The proposed algorithm is experimented on various Brodatz and Vistex textures and the results show the efficiency of segmentation.
\end{abstract}

\section{KEYWORDS}

Texture, Segmentation, Image enhancement, Noise removal.

\section{INTRODUCTION}

Texture can be thought of as a replicated pattern and is portrayed on the surface of an object $[15,16,17]$. Segmentation is a basic low-level operation on images used to divide textures into a single texture type. In order to separate the textures the spatial information for each texture must be preserved. The group of connected pixels that share a common feature form a homogenous region. This feature could be motion, brightness, depth, color, texture etc. For example, the grey level thresholding algorithm done manually does not provide the spatial information for each texture $[12,14]$ and can lead to incorrect segmentation result. The segmentation techniques can be classified into two types based on the number of images known in advance, supervised [1-3] or unsupervised [4-6]. Since in most of the practical applications the number of images is generally unknown, the unsupervised approach is considered to be more beneficial. There are three main groups of image texture segmentation that come under the above two techniques. They are edge-based, cluster-based and region based methods [7]. Image segmentation is a common and important task aimed at extracting objects that can be subsequently measured or analyzed. This is in particular useful when looking for defects in materials or for pathological cells in a biological tissue. In many cases, the background and the objects themselves are non-uniform and made of so-called textures. A challenging problem concerns the automatic extraction (or segmentation) of various textures present in the same image.

The edge-based segmentation exploits spatial information by identifying the edges in an image, where the edges are the discontinuities in the homogeneity criterion for segments. Edges in a 
Signal \& Image Processing : An International Journal (SIPIJ) Vol.4, No.1, February 2013

given depth map are defined by the points where differences in the local surface properties cross a given threshold value. The local surface properties commonly used are gradients, surface normal's, principal curvatures or higher order derivatives. Detection of edges on the texture image can lead to noisy and broken edges and therefore segmentation process becomes more difficult [13]. The edge detection methods are very sensitive to noise in the range data because these methods respond to abrupt changes in the homogeneity property used for segmentation. The available information is not optimally utilized because only the measurements near the edges are used to make major decisions. In most of the situations the edges do not form closed boundaries and the task is more complicated to make correct grouping decisions there by resulting in under or over segmentation. Many researchers reported the typical variations on the edge-based segmentation techniques in $[8,9,10,11]$.

In computer vision, edge detection is an important task. There are a wide range of applications from segmentation to pattern matching. It decreases the computational complexity of the image allowing more costly algorithms like object matching, object recognition, object registration, or surface reconstruction from stereo images to be used. Mathematical morphology (MM) is one of the approaches which is used for image enhancement problem in digital images .Such operators consist in accordance to some proximity criterion, in choosing for each point of the analyzed image, a new grey level between two patterns (primitives) [22], [23]. There are a few methodologies based on MM, which are used for contrast enhancement and segmentation of poor illumination images. One of the most common algorithms in image processing to enhance dark regions is the use of nonlinear functions like logarithm or power functions or a method that

Works well in the frequency domain is the homomorphic filter. Histogram equalization is also commonly used for enhancement but the global properties of the image cannot be properly applied in a local context and also it produces poor performance in detail preservation.Edge characterize object boundaries and therefore useful for segmentation and identification of objects in scenes. Edge points are pixel locations of abrupt gray level changes. The basic idea underlying most edge-detection techniques is the computation of a local derivative operator.

Many segmentation algorithms have been proposed in the literature, which use mathematical/theoretical tools, such as genetic algorithms, fuzzy logic, neural networks (NN), pattern recognition, wavelet, and so forth, or based on unique frameworks, such as active contour models (ACM), watershed, thresholding and so forth. The intervention of a human operator in practice is often necessary because using fully automatic methods sometimes would fail and produce wrong results. To identify the patterns used in the interaction for the segmentation of medical images and to develop qualitative criteria for the evaluation of interactive segmentation methods, a review of computational algorithms involving human-computer interaction in image segmentation has been made in [20]. This survey has taken into account the type of information provided by the user, how this information affects the computational part of the method and the purpose of interaction in the segmentation process for the comparison and classification of a number of human-machine dialog methods. Thresholding method is a relative fast and simple technique. A survey of thresholding methods with a view to assess their performance when applied to aerial images has been made recently in [21]. Some image examples are taken from oceanographic applications in this work.

The organization of this paper is as follows: Section 2 gives the related work; introduction to mathematical morphology is given in section 3, the proposed method and algorithm described in section 4. Experimental results of texture segmentation are shown in the section 5 and section 6 gives the conclusions.

\section{MATHEMATICAL MORPHOLOGY}

Morphology used in the context of mathematical morphology as a tool for obtaining image components that are useful in the description and representation of region shape, such as 
Signal \& Image Processing : An International Journal (SIPIJ) Vol.4, No.1, February 2013

skeletons, boundaries. The mathematical morphology is a set theory. As such, morphology offers a powerful and unified approach to various image processing tasks. Sets in mathematical morphology represent objects in an image. Binary images can be represented as sets whose components are $\mathrm{Z}^{2}$, where each element of a set is a 2-D vector whose coordinates are the (x,y). Gray scale digital images can be represented as sets whose components are in $Z^{3}$. In this case, two components of each element of the set refer to the coordinates of pixel, and the third corresponds to its discrete gray-level value. An essential part of the morphological operations is the structuring element used to probe the input image and is itself a binary image. A structuring element is a matrix consisting of only 0 's and 1 's that can have any arbitrary shape and size, drawing conclusions on how this shape changes the input image. The pixels with values of 1 define the neighborhood in the structuring element used for the process of morphological operations. There are basically two morphological operations known as Dilation, Erosion.

Dilation: Erosion removes pixels on object boundaries whereas dilation adds pixels to the boundaries of objects in an image. The number of pixels removed or added from the objects in an image depends on the shape and size of the structuring element used to process the image. In the morphological erosion and dilation operations, the state of any given pixel in the output image is determined by applying a rule to the corresponding pixel and its neighbours in the input image.

Erosion: The erosion operator has the opposite effect. Now the result is only one, if the mask is completely within the object. Objects smaller than the mask disappears completely. The number of pixels removed or added from the objects in an image depends on the shape and size of the structuring element used to process the image. In the morphological erosion and dilation operations, the state of any given pixel in the output image is determined by applying a rule to the corresponding pixel and its neighbours in the input image. The rule used to process the pixels defines the operation as erosion or dilation.

Using the elementary dilation and erosion operations, two useful operations were developed to work on the form of objects. The morphological opening of an image is erosion followed by dilation, using the same structuring element for both operations. The related operation, morphological closing of an image, is the reverse: it consists of dilation followed by erosion with the same structuring element. Closing and opening are two important operators from mathematical morphology. They are both derived from the fundamental operations of dilation and erosion. Like those operators, they are normally applied to binary images, although there are also gray level versions. Opening is the dual of closing, i.e. opening the foreground pixels with a particular structuring element is equivalent to closing the background pixels with the same element. When an erosion operation is applied to an image, it eliminates the small objects present in the image. Here there is a demerit that all the remaining objects in the image shrink in size. To avoid this affect, we apply dilation operation for the eroded image with the same structuring element. This combination of operation is called opening operation.

The field of mathematical morphology has one more important operator called closing. Like its dual operator opening, it can be derived from the basic operations of erosion and dilation. Like those operators, it is generally applied to binary images. Closing and dilation are similar in some ways that it tends to extend the boundaries of bright (foreground) regions in an image (and shrink background color holes in such regions), but it does not destroy the original boundary shape. As with other morphological operators, the exact operation is determined by a structuring element. The effect of the operator is to preserve background regions that have a similar shape to this structuring element, or that can completely contain the structuring element, while eliminating all other regions of background pixels.

One of the applications of dilation is to fill in small background color holes in images, e.g. 'pepper noise'. One of the difficulties with doing this, however, is that the dilation will also distort all regions of pixels indiscriminately. By performing erosion on the image after the dilation, i.e. a closing, this effect is reduced to some extent. The effect of closing can be quite easily visualized. 
Signal \& Image Processing : An International Journal (SIPIJ) Vol.4, No.1, February 2013

Imagine the structuring element sliding around outside each foreground region, without altering its orientation. For any background boundary point, if the structuring element can be made to touch that point, with no part of the element being inside a foreground region, then that particular point remains background. If this is not possible, then the pixel is set to foreground. After the closing has been carried out the background region will be such that the structuring element can be made to cover any point in the background with no part of it also covering a foreground point, and so further closings will have no effect. This property is known as idempotence.

\section{METHODOLOGY}

Texture segmentation divides an image into a set of disjoint regions based on texture properties. To deal the issues of segmentation the present paper divided the entire process of segmentation into ten steps.

STEP 1: Read the gray scale image. Perform upsampling i.e. interpolation on the image. The area of the image is doubled and zeros are padded in between each element and these zeros are now replaced with the average of the neighbouring elements in a particular fashion. Row element padded zeros are replaced by averaging preceding and next row elements and similarly column elements padded zeros are replaced by averaging preceding and next column elements.

STEP 2: Perform median filtering on the image obtained from step 1.

STEP 3:Perform decimation or down sampling on the image obtained from step 2 i.e. the area of the image is reduced by half and the previously padded elements in step 1 are removed, making the image area again same as original image.

STEP 4: Contrast limited adaptive histogram equalization is performed on the image obtained in step 3.

STEP 5: Define a line structuring element of particular size and rotate it from 0 to 180 degrees with an interval of 15 degrees. Obtain bottom hat transform of the step 4 output image using the structuring element. 12 images are obtained as output.

STEP 6: Add all the 12 images obtained as output in step 5. This will produce high quality image in which local objects are identified more clearly.

STEP 7: Perform averaging filter operation on the image obtained in step 6. The output at this step will produce noise, which will be useful in noise removal. The size of the averaging filter is $40 \mathrm{x} 40$.

STEP 8: The obtained noise in step 7 is eliminated by subtracting the outputs of step 6 and step 7 i.e. bottom hat output and averaging filter output.

STEP 9: The obtained image is contrast stretched to identify the local objects clearly using special operators.

STEP 10: Segmentation is performed on the final image obtained in step 9 by using Laplacian of Gaussian (LOG) operator of size $5 \times 5$ to extract the required objects.

\section{RESULTS AND DISCUSSION}

The proposed algorithm has been tested on brodatz and vistex textures of size $256 x 256$. The results are shown in Fig.1 to Fig.2. Stepwise outputs. Fig. 3 and Fig.4 show the original images from database and their respective final segmented outputs. The (a) images of Fig.4 show the final outputs of proposed method, (b) images of Fig.4 show the outputs of the method in [18] and (c) images of Fig.4 show the outputs of the method in [19]. The connectivity of the pixels in the edges is well established in the outputs of the proposed method when compared to the other two 
methods as shown in Fig.4. The proposed method outputs show that the contrast is more when compared to the outputs of the methods in [18], [19]. The results obtained are satisfactory. The proposed method reduces the noise if present and segments the image in 15 different directions. The proposed method works well for dark textures as the method involves CLAHE.

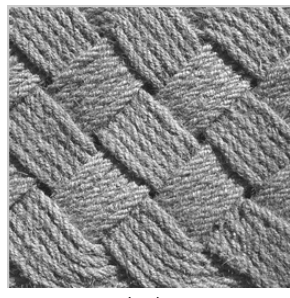

(a)

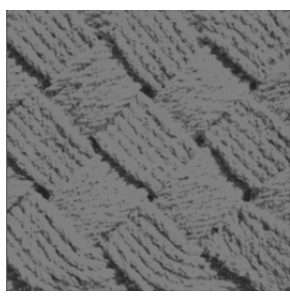

(b)

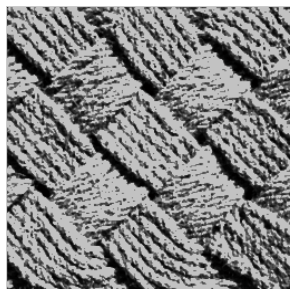

(c)

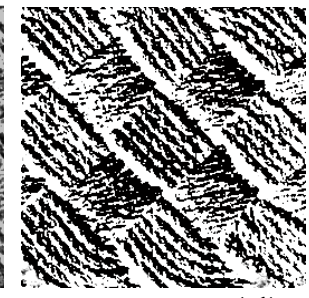

(d)

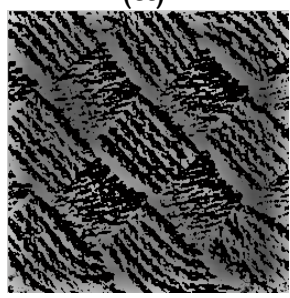

(e)

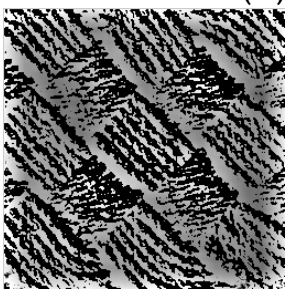

(f)

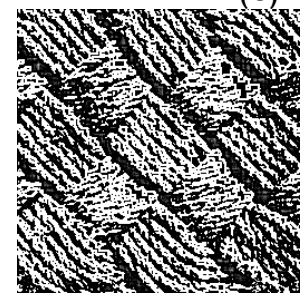

(g)

Figure 1.(a) Original Fabric11 (b) Step 2 output (c) Step 4 (d) Step 5 (e) Step 8 (f) Step 9 (g) Final Segmented Output.

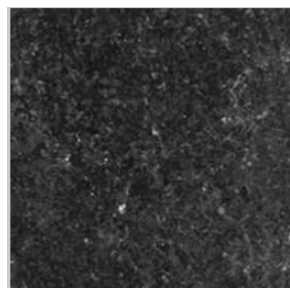

(a)

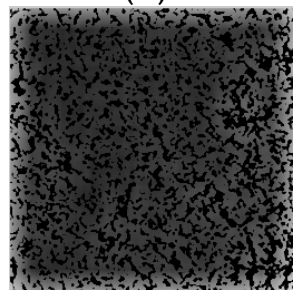

(e)

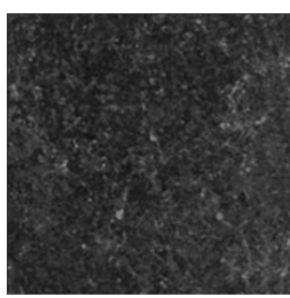

(b)

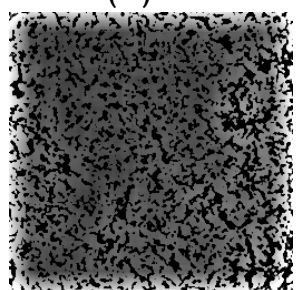

(f)

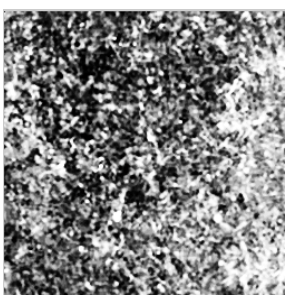

(c)

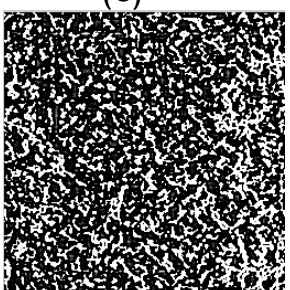

(g)

Figure 2.(a) Original blackpearl (b) Step 2 output (c) Step 4 (d) Step 5 (e) Step 8 (f) Step 9 (g) Final Segmented Output.

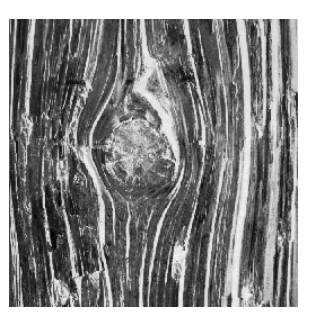

(a)

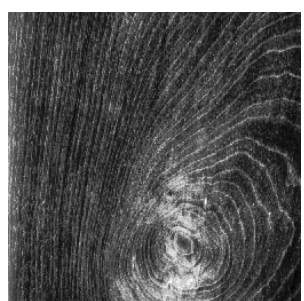

(b)

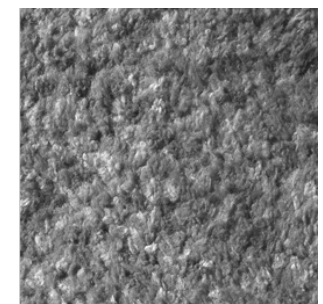

(c)

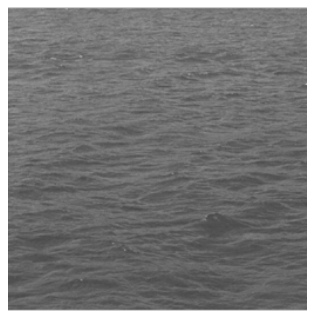

(d)

Figure 3.Original images (a) D72 (b) 69 (c) Fabric 15 (d) Water 3 

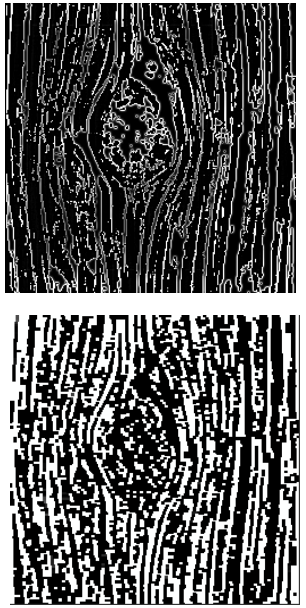

(b)

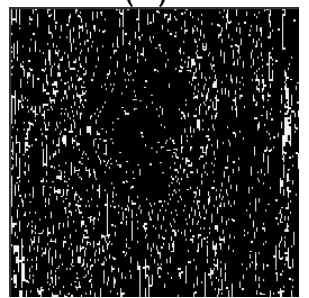

(c)
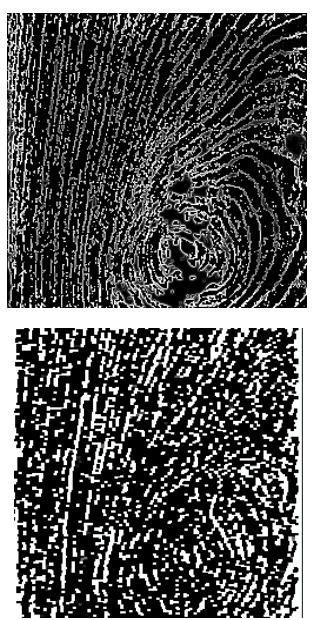

(b)

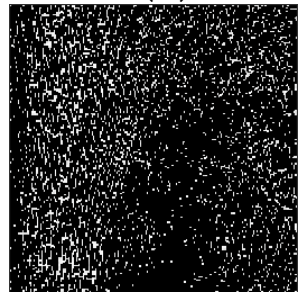

(c)
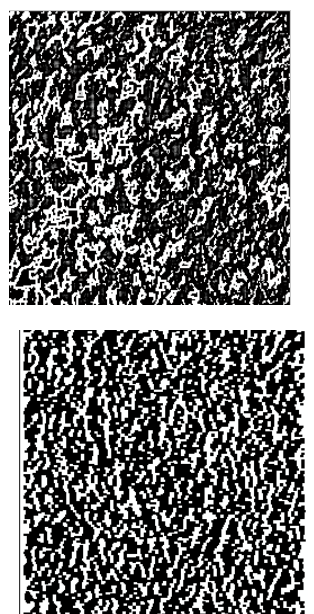

(b)

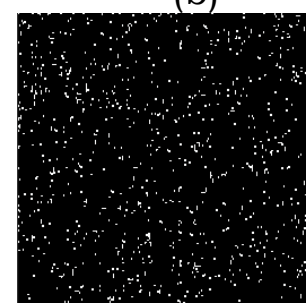

(c)
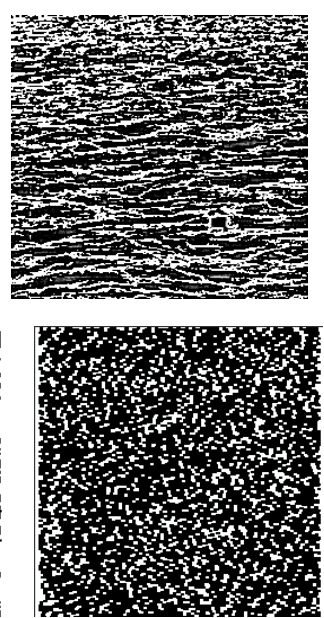

(b)

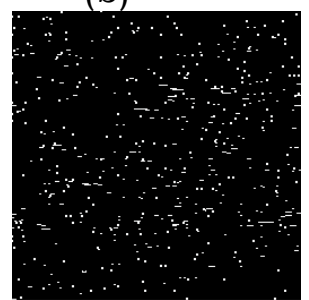

(c)

Figure 4. (a) Corresponding outputs of figure 3 for the proposed method, (b) Corresponding outputs of figure 3 for the method in [18], (c) Corresponding outputs of figure 3 for the method in [19]

\section{Conclusions}

The proposed algorithm uses different ways to enhance, detect and segment the image details. CLAHE improves the contrast of sharp details in both bright and dark regions due to its window based nature. It produces better results compared to global based methods such as histogram equalization. Bottom hat morphological transformation with 12 line structuring elements of size 14 and degrees from 0 to 180 has been performed. This method extracts all the dark objects from the background of the image. A background noise subtraction process is then performed to remove noise in the images. In order to segment the image, laplacian of Gaussian operator is applied, the importance of this operator is it removes the noise if present and then identifies the segments in the image. The segmentation results are satisfactory and the algorithm does not involve complex computations. The proposed algorithm does segmentation along with enhancement.

\section{ACKNOWLEDGEMENTS}

The authors would like to express their gratitude to Sri K.V.V. Satya Narayana Raju, Chairman, and K. Sashi Kiran Varma, Managing Director, Chaitanya group of Institutions for providing necessary infrastructure. Authors would like to thank anonymous reviewers for their valuable comments and SRRF members for their invaluable help. The authors would like to express their gratitude to Dr.G.Tulsi Ram Das, Vice chancellor, JNTU Kakinada. 
Signal \& Image Processing : An International Journal (SIPIJ) Vol.4, No.1, February 2013

\section{REFERENCES}

[1] D.Dunn, W. E. Higgins and J. Wakeley, Texture segmentation using 2-D Gabor elementary functions, IEEE Trans. Pattern Analysis Mach. Intell. 16(2), 130- 149, 1994.

[2] Y. M. Zhu and R. Goutte, Analysis and comparision of space/spatial - frequency and multi scale methods for texture segmentation, Opt. Eng. 34(1), 269-282(1995).

[3] M. Unser, Texture classification and segmentation using wavelet frames, IEEE Trans. Image Process. 4(11), 1549-1560, 1995.

[4] J. Mao and A. K. Jain, Texture classification and segmentation using multiresolution simultaneous autogressive models, Pattern Recognition 25(2), 173-188,1992.

[5] Y. Hu and T. J. Dennis, Textured image segmentation by context enhance clustering, IEEE Proc. Vis. Image Signal Process. 141(6), 413-421, 1994.

[6] J. L. Chen and A. Kundu, Unsupervised texture segmentation using multichannel decomposition and hidden Markov models, IEEE Trans. Image Process.4(5), 603-619,1995.

[7] R.C. Gonzalez, and R.E.Wood,.. Digital Image Processing., Wesley Publishing Company, pp. 458461,2002.

[8] O.R.P.Bellon, A.I. Direne, and L. Silva, Edge detection to guide range image segmentation by clustering techniques. In: International Conference on Image Processing (ICIP '99), Kobe, Japan, pp. 725-729, 1999.

[9] X. Jiang, A. Hoover, G. Jean-Baptiste, D. Goldgof, K. Boywer, and H. Bunke, A methodology for evaluating edge detection techniques for range images. In: Proc. Asian Conf. Computer Vision, pp. 415-419, 1995.

[10] A.D. Sappa, and M. Devy, Fast range image segmentation by an edge detection strategy. In: Third International Conference on 3-D Digital Imaging and Modeling, pp. 292-299, 2001.

[11] M.A. Wani and H.R. Arabnia, Parallel edge-regionbased segmentation algorithm targeted at reconfigurable multiring network. Journal of Supercomputing 25(1), pp. 43-62, 2003.

[12] C.E. Honeycutt and R. Plotnick, Image analysis techniques and gray-level co-occurrence matrices (GLCM) for calculating bioturbation indices and characterizing biogenic sedimentary structures, Computers \& Geosciences 34, pp. 1461-1472, 2008.

[13] S. Zheng, J. Liu and J.W. Tian, A new efficient SVMbased edge detection method, Pattern Recognition Letters 25, pp. 1143-1154, 2004.

[14] S.Kezia, I.Shanti Prabha, V.VijayaKumar, Innovative Segmentation Approach Based on LRTM, IJSCE, Vol.2, Iss.5, November 2012.

[15] Saka.Kezia, Dr.I.Shanti Prabha, Dr.V.Vijaya Kumar, A New Texture Segmentation Approach for Medical Images, IJSER, Vol. 4, Iss.1, January-2013.

[16] M. Joseph Prakash, Saka. Kezia, I. Santi Prabha, V. VijayaKumar, A novel approach for texture segmentation based on rotationally invariant patterns, IJCES,Jan 2013.

[17] M. Joseph Prakash, V.Vijayakumar, A new texture based segmentation method to extract object from background, GJCST,Graphics \& Vision,Vol.12, Iss.15, Dec 2012.

[18] B. V.Ramana Reddy, M.Radhika Mani,and V.Vijaya Kumar, A Random Set View of Texture Segmentation, IJSIP, vol.1,iss.1,pp.24-30,2010.

[19] M.Joseph Prakash, S.Kezia, B.V. Ramana Reddy, "An approach for texture segmentation based on random field model and wavelet fusion”, IJSIP, Vol. 1, Iss.3-2010, pp.183-189.

[20] Olabarriaga, S. D., \& Smeulders, A.W. M, Interaction in the segmentation ofmedical images: A survey. Medical Image Analysis, 5(2), 127-142, 2001.

[21] Marcello, J., Marques, F., \& Eugenio, F, Evaluation of thresholding techniques applied to oceanographic remote sensing imagery. SPIE, 5573, 96-103, 2004.

[22] I. R. Terol-Villalobos, "A multiscale contrast approach on Morphological connected contrast mappings" Opt. Eng., vol. 43, no. 7, pp. 1577-1595, 2009.

[23] F. Meyer and J. Serra, “Contrast and Activity Lattice,” Signal Processing, vol. 16, pp. 303-317,1989. 
Authors

M.Joseph Prakash received the B.E (ECE) Degree from Andhra University in 1996 and received his M. Tech. in Computer Science from JNUCE, JNT University, Kukatpally,Hyderabad, India in 2003. He is having nearly 16 years of teaching and industrial experience. He handled various positions Professor\&HOD,I/C Principal, Co-coordinator, currently with GIET, IT Dept., Rajahmundry, Andhrapradesh, India. He is pursuing his Ph.D from JNT University, Kakinada in Computer Science under the guidance of Dr. V. Vijaya Kumar. He is a life member of Computer Society of India, ISTE, Red Cross Society and he is a member of SRRF-GIET, Rajahmundry. He has presented 9 papers in various National, Inter National conferences proceedings.

Vakulabharanam Vijaya Kumar received integrated M.S. Engg. , degree from Tashkent Polytechnic Institute Associate Professor and taught courses for M.Tech students. He has been working as Dean Computer sciences and Head Srinivasa Ramanujan Research Forum-GIET, Rajahmundry, Affiliated to JNT University, Kakinada. His research interests include Image Processing, Pattern Recognition, Network Security, Steganography, Digital Watermarking, and Image retrieval. He is a life member for CSI, ISC, ISTE, IE (I), IRS, ACS, CS and Red Cross. He has published more than 100 research publications in various National, Inter National conferences, proceedings and Journals.

Dr.A.Vinaya Babu received $\mathrm{ME}(\mathrm{ECE}), \mathrm{M}$.Tech,(CSE) ,PhD degree inComputer Science. He is Principal of JNTUHCE and Professor of CSE at JNTUHCE, JNTUH. He has a total of 25 years of experience in Teaching, Has a total of 40 publications in National and International Journals. He Received the Academic Excellence Award and Jewel of India Award. Is a member of many professional bodies like IEEE, IETE, ISTE, CSI. His area of interests Image Processing, Algorithm, Information Retrieval and Data mining, Computational Models, Computer Networks, and Computer Architecture
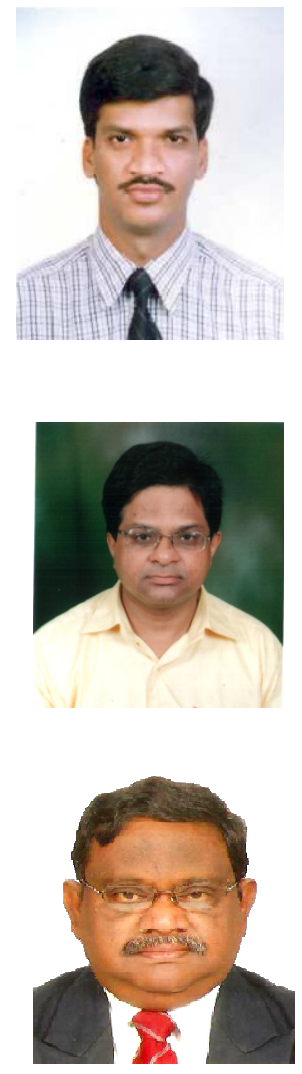